\title{
QUELQUES TRAITS BIOÉCOLOGIQUES DES ALOSES DU MAROC ; UN PATRIMOINE CULTUREL ET SOCIO-ÉCONOMIQUE RÉCEMMENT DISPARU.
}

\author{
R. SABATIÉ $(1,2)$, J.L. BAGLINIÈRE (2)
}

(1) ENSAR, Département Halieutique, 65, rue de St Brieuc, CS 84215, 35042 RENNES Cedex, France.

(2) UMR INRA-ENSAR, Ecologie et qualité des Hydrosystèmes continentaux, 65, rue de St Brieuc, CS 84215, 35042 RENNES Cedex, France.

\section{RÉSUMÉ}

Le Maroc abritait dans ses plus grands fleuves les populations d'aloses ( $A$. alosa et A. fallax) les plus méridionales de l'aire de distribution, mais diverses pollutions et les constructions de barrages ont considérablement réduit leur effectif dont la production atteignait $700 \mathrm{t}$ dans les années 70 . Seul l'oued Sebou et son affluent l'Ouerrha, offraient encore récemment jusqu'en 1991, des lieux de frai accessibles.

Entre 1978 et 1988, 1223 aloses ont été étudiées. Elles ont montré des caractéristiques morphologiques (biométriques et méristiques) et écobiologiques particulières, beaucoup plus nettes chez la grande alose, qui ont été comparées à celles de populations septentrionales. II est apparu que la valeur modale du nombre de branchiospines des aloses vraies (110) est la plus faible de l'aire de distribution. Cette particularité peut résulter de plusieurs facteurs modulateurs (température, taille des proies consommées, compétition interspécifique en mer). Egalement, la plus faible valeur des moyennes de caractères méristiques (rayons de nageoires, scutelles) peut exprimer la conservation de caractères archaïques qui ont été récemment soulignés par une étude génétique des aloses feintes. Les grandes aloses femelles ont un âge moyen de 5,8 ans contre 5 ans chez les mâles ; la particularité des écailles est la présence de marques hivernales cicatricielles et d'une marque de crue, souvent plus visible chez les juvéniles. Les performances de croissance remarquable en longueur et en poids des aloses vraies sont probablement liées à la richesse trophique des eaux côtières qu'elles colonisent (présence d'un upwelling). Enfin, le flux migratoire au Maroc est beaucoup plus étalé dans le temps (6 mois contre 4$)$ et décalé vers l'automne-hiver.

La grande alose qui était un patrimoine d'intérêt également socio-culturel et scientifique a malheureusement disparu depuis 1992 des eaux marocaines. 


\title{
SOME ECOBIOLOGICAL TRAITS IN MORROCAN SHADS ; A CULTURAL AND SOCIO-ECONOMIC VALUE INTEREST WHICH HAS DISAPPEARED.
}

\begin{abstract}
Morrocan Allis Shad and Twaite Shad were the most southern populations of the Eastern Atlantic but the distribution area has been considerably reduced - $700 \mathrm{mt}$ were catched in Sebou's fisheries until 70's - by alterations resulting from aquatic pollution, hydro-electric impoundments and industrial installations. Along the Atlantic coast, the Sebou river and its main tributary, the Ouerrha, were the only remaining accessible spawning grounds for spawners until 1991. In 1992 an estuary dam stopped the upriver migration of shads ; a fish pass was buildt, but seemed unsuccessful.
\end{abstract}

Biological and morphological characteristics were studied from a sample of more than 1223 shads, caught between 1978 and 1988. Morrocan populations have specific features and characteristics (very sharp in Allis Shad) as compared to these of northern populations. The Alosa alosa population share a lower modal number of gillrakers (110) whose origine is probably coming from some modulating factors (temperature, size of preys, interspecific trophic competition in the sea). The lower average number of some meristic characteristics (fin ray, scutes) probably reflects the plesiomorphic state of characters. This ancestral state has been emphasized by electrophoretic analysis of twaite's shad proteins. The average age of female and male is 5,8 and 5 years respectively and the adult scales showed characteristics cicatricial winter rings while a flood mark is present in some juveniles scales. The higher growth of Sebou shads results from the presence of an upwelling zone in sea where trophic resources were abundant. The migration period is longer and starts earlier in Sebou River (December) than in Loire (March).

Presently, the Allis Shad has disappeared from morrocan waters since 1992, while it was a great cultural, socio-economic and scientific value interest.

\section{INTRODUCTION}

Le Maroc abritait dans ses plus grands fleuves, les populations d'alose vraie ou grande alose (Alosa alosa Linné, 1758) et d'alose feinte (Alosa fallax Lacépède, 1803) les plus méridionales de l'aire de distribution de l'Atlantique Est qui s'étend vers le Nord jusqu'au sud de la Norvège (Figures 1,12 ). Dès le XV| ${ }^{\text {eme }}$ siècle, l'exploitation des aloses dans ce royaume était très activement menée par les portugais et plusieurs pêcheries renommées (Azemmour, Kénitra) alimentaient les marchés intérieurs et exportaient même leurs produits salés et séchés.

Au début du siècle, WATIER (1918) estimait que la production globale des oueds (dont la Moulouya sur la Méditerranée) atteignait $1000 \mathrm{t}$, mais dès 1929, les barrages se sont multipliés sur les cours d'eau comme aussi la forte dégradation générale des habitats côtiers et continentaux (AZEROUAL et al., 2000). Aussi, même si dans les années 70 on pêchait encore $700 \mathrm{t}$ d'aloses dans l'oued Sebou et le proche littoral, plusieurs bassins versants avaient déjà perdu leurs populations d'aloses. Dès 1980, ce fleuve et son principal affluent l'Ouerrha, abritaient les derniers sites de frai pouvant accueillir les géniteurs.

Depuis, les deux espèces ont progressivement disparu de cet oued où l'implantation d'un barrage estuarien en 1992 a définitivement condamné la migration de la grande alose (Figure 2). Aucune mesure rigoureuse n'a été entreprise pour limiter l'impact de l'obstacle 
sur les migrations puisque la passe à poisson construite n'a pas donné lieu à un programme de suivi de l'efficacité de l'ouvrage.

La localisation méridionale de ces populations exploitées, affectées par divers aménagements du réseau hydrographique, offrait l'intérêt de pouvoir compléter la connaissance de leur écobiologie, de réactualiser les travaux plus anciens qui leur avaient été consacrés (FURNESTIN, 1952 ; FURNESTIN et VINCENT, 1955 , 1958 ; LAHAYE et al., 1963) et de participer aux études qui se développaient sur les populations d'aloses européennes (CASSOU-LEINS et CASSOU-LEINS, 1981 ; DOUCHEMENT, 1981 ; EIRAS, 1981 ; MENESSON-BOISNEAU et BOISNEAU, 1990). Pour ces raisons, une étude détaillée de la morphologie et de bioécologie a été entreprise pendant plusieurs années (1978-1988) sur les aloses du Sebou et plusieurs des populations résiduelles (SABATIÉ, 1990, 1993). Une hybridation sporadique entre les deux espèces a été relevée et semblait liée à la précocité et/ou à la sévérité de l'étiage. Globalement, ces aloses présentaient des caractéristiques biologiques originales qui sont analysées et comparées au stade adulte par rapport aux populations septentrionales.

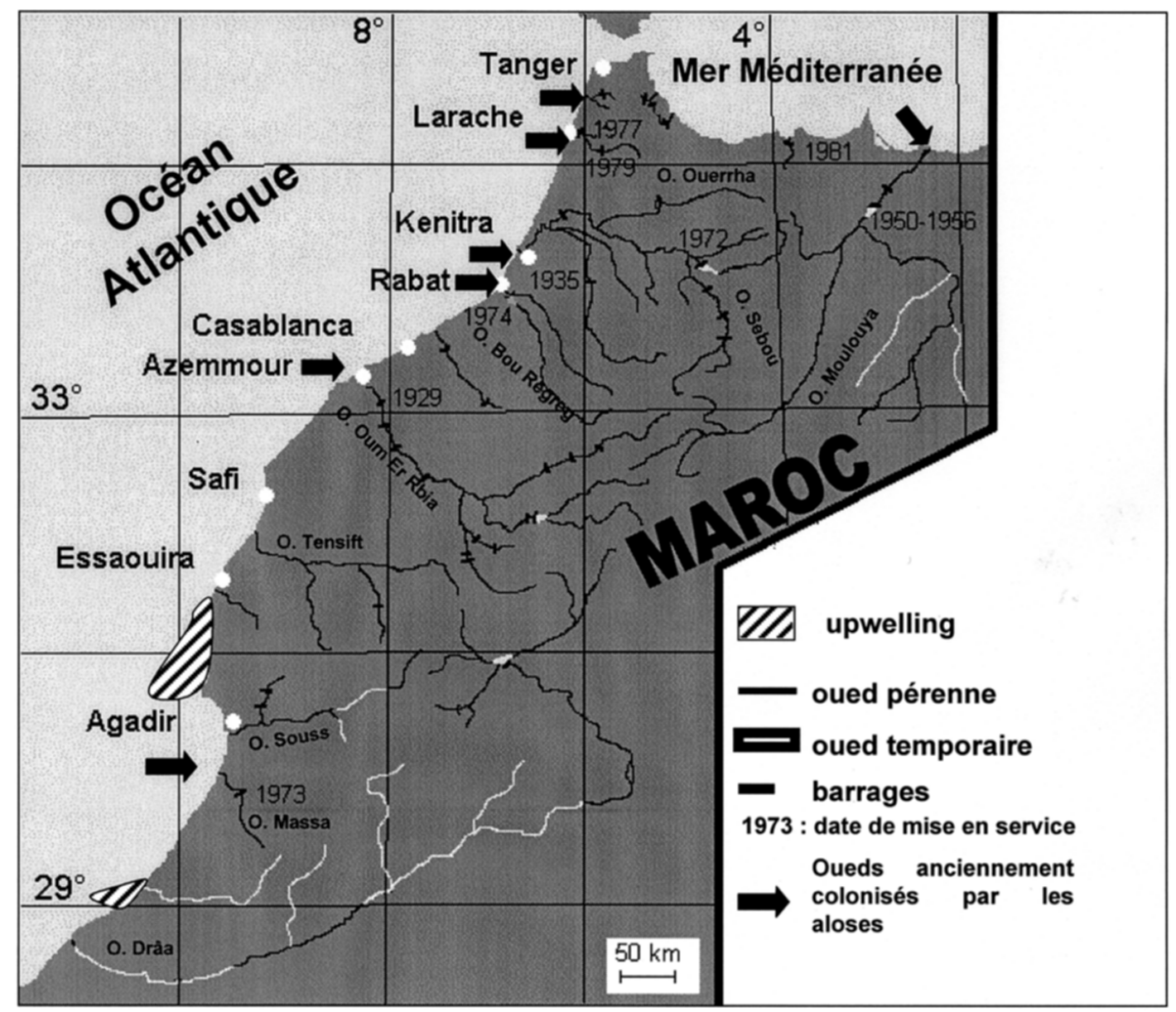

Figure 1

Distribution ancienne des populations d'aloses le long des côtes marocaines et localisation des principaux barrages (d'après SABATIÉ, 1993).

Figure 1

Historic distribution of shad populations along the Moroccan coast and the main localisation of dams along the rivers (from SABATIÉ, 1993). 


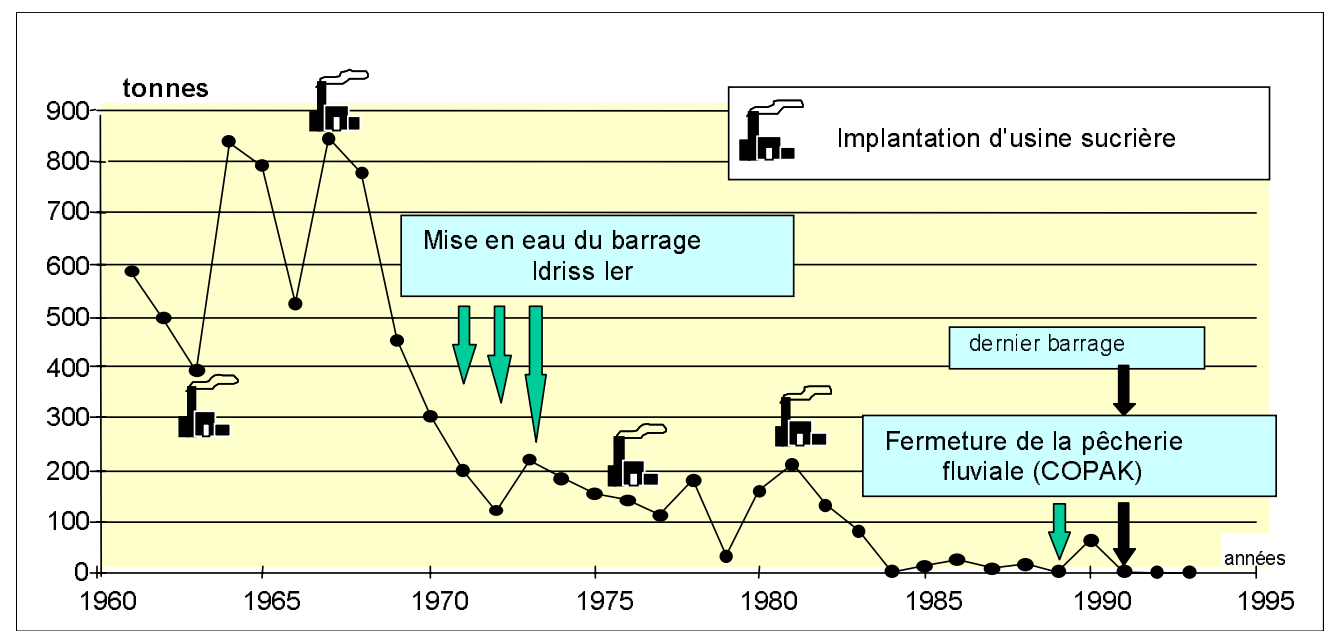

\section{Figure 2}

Captures annuelles d'aloses vraies ; pêcheries maritime (Mehdia) et fluviale (COPAK) de l'oued Sebou en relation avec les aménagements et les pollutions (d'après Sabatié, 1993).

\section{Figure 2}

Annual catches of Allis Shad from the sea (port of Mehdia) and river (co-operative COPAK) fisheries of Sebou River as related to dams construction and pollution sources (from Sabatié, 1993).

\section{MATÉRIEL ET MÉTHODES}

Le bassin versant du Sebou couvre $40000 \mathrm{~km}^{2}$ et la longueur de l'axe fluvial est de $400 \mathrm{~km}$. Le débit moyen oscille autour de $130 \mathrm{~m}^{3} / \mathrm{s}$, mais peut atteindre $5000 \mathrm{~m}^{3} / \mathrm{s}$. L'échantillon analysé était constitué de poissons capturés durant plusieurs années au filet maillant ou tramail, fixe ou dérivant, par les pêcheurs professionnels, en mer et dans les fleuves ; 729 grandes aloses, 451 aloses feintes et 43 aloses hybrides débarquées en criée ont été analysées. La sélectivité des maillages utilisés écartait des captures les poissons les plus petits, immatures âgés au plus d'un an, qui circulent dans l'estuaire, mais les engins utilisés permettaient la capture de petits géniteurs migrant tel un mâle d'alose feinte qui mesurait $24 \mathrm{~cm}$. Les mesures en longueur totale ont été faites au $1 / 2 \mathrm{~cm}$ inférieur et le poids frais relevé au g près.

L'analyse des données a été réalisée par les tests paramétriques $F$ de SNEDECOR et $t$ de STUDENT pour comparer variances et moyennes des échantillons. Pour ces analyses comparatives il a été vérifié (études intercohortes ou interannuelles) que les données provenant des autres fleuves pouvaient être aussi regroupées et que les conditions de capture étaient sensiblement identiques. Ces aloses, pêchées en estuaire ou plus en amont, mais avant la reproduction, possèdent des estomacs pratiquement vides, des ovaires dont le stade de développement vitellogénique est bloqué et un état de condition encore non affecté par les efforts migratoires.

L'analyse électrophorétique des protéines d'échantillons congelés (> $1 \mathrm{ml})$ du sang, du foie et du muscle a permis récemment de caractériser génétiquement un échantillon de 21 aloses feintes. Les techniques utilisées à partir de l'échantillon de foie pour identifier les loci sont l'électrophorèse sur gel d'amidon (MPI ou Mannose Phosphate Isomérase : ADH ou Alcool Déshydrogénase) ou par isoélectrofocalisation sur gel de polyacrilamide (HBA ou chaîne alpha de l'Hémoglobine : ADA ou Adénosine Déshydrogénase : PX ou Protéine 
plasmatique non identifiée) et sont décrites par ALEXANDRINO (1996). Les calculs d'analyses des fréquences alléliques et des distances génétiques de NEI (1978) sont réalisés à l'aide du logiciel BIOSYS 1.7 et PHYLIP 3.5. L'arbre représente les différentiations quantitatives réelles en terme de distance génétique entre les populations et doit être interprété comme tel. Chaque taxon représente un « outgroup » par rapport aux autres.

\section{RÉSULTATS - DISCUSSION}

\section{Méristique}

L'étude de l'évolution du nombre de branchiospines chez les aloses juvéniles et chez les géniteurs âgés de 3 à 8 ans montre que l'accroissement des peignes branchiaux est rapide durant les premiers mois et que la ségrégation entre les trois taxons peut être déjà réalisée. Chez les adultes, malgré la difficulté à discriminer quelques individus en limite de séparation des groupes taxonomiques (Figure 3), on peut constater que le nombre de branchiospines est stabilisé durant leur croissance (corrélations non significatives) (Figure 4) et qu'une valeur moyenne du caractère peut être attribuée alors pour chacun des taxons. Une étude comparative de ce caractère sur l'aire de répartition a mis en évidence de façon nette chez la grande alose et les hybrides marocains, la forte variabilité du caractère entre les populations (hétérogénéité des variances) et la valeur moyenne relativement faible du nombre de branchiospines (Tableau I). Ce trait est moins marqué chez les aloses feintes.

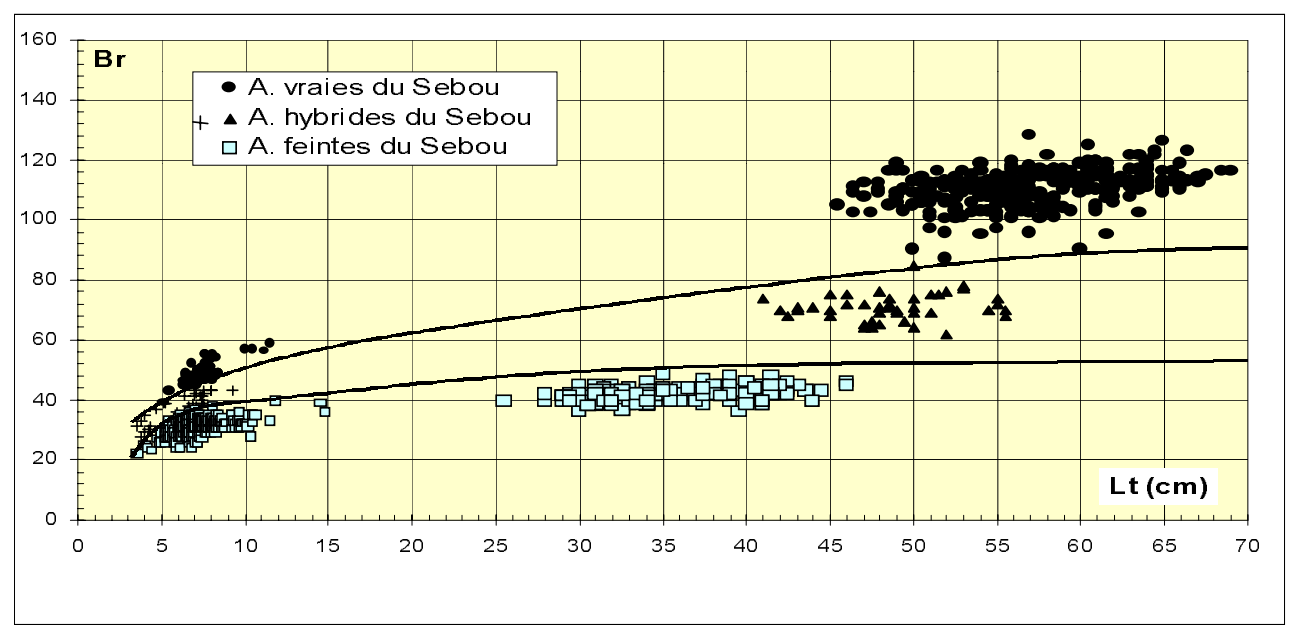

Figure 3

Distribution du nombre total de branchiospines $(\mathrm{Br})$ en fonction de la longueur totale (Lt) chez les populations d'aloses du Sebou, sexes confondus. Des analyses discriminantes entre individus placés aux limites des trois groupes taxonomiques ont permis d'extrapoler des courbes de tri sur la base des échantillons recueillis.

\section{Figure 3}

Relation of total gillraker number $(\mathrm{Br})$ to total length $(\mathrm{Lt})$ in both sex of Allis Shad from Sebou River. Discriminant analysis on fish located on border between the three taxonomic groups allowed to drawn a discriminant curve. 


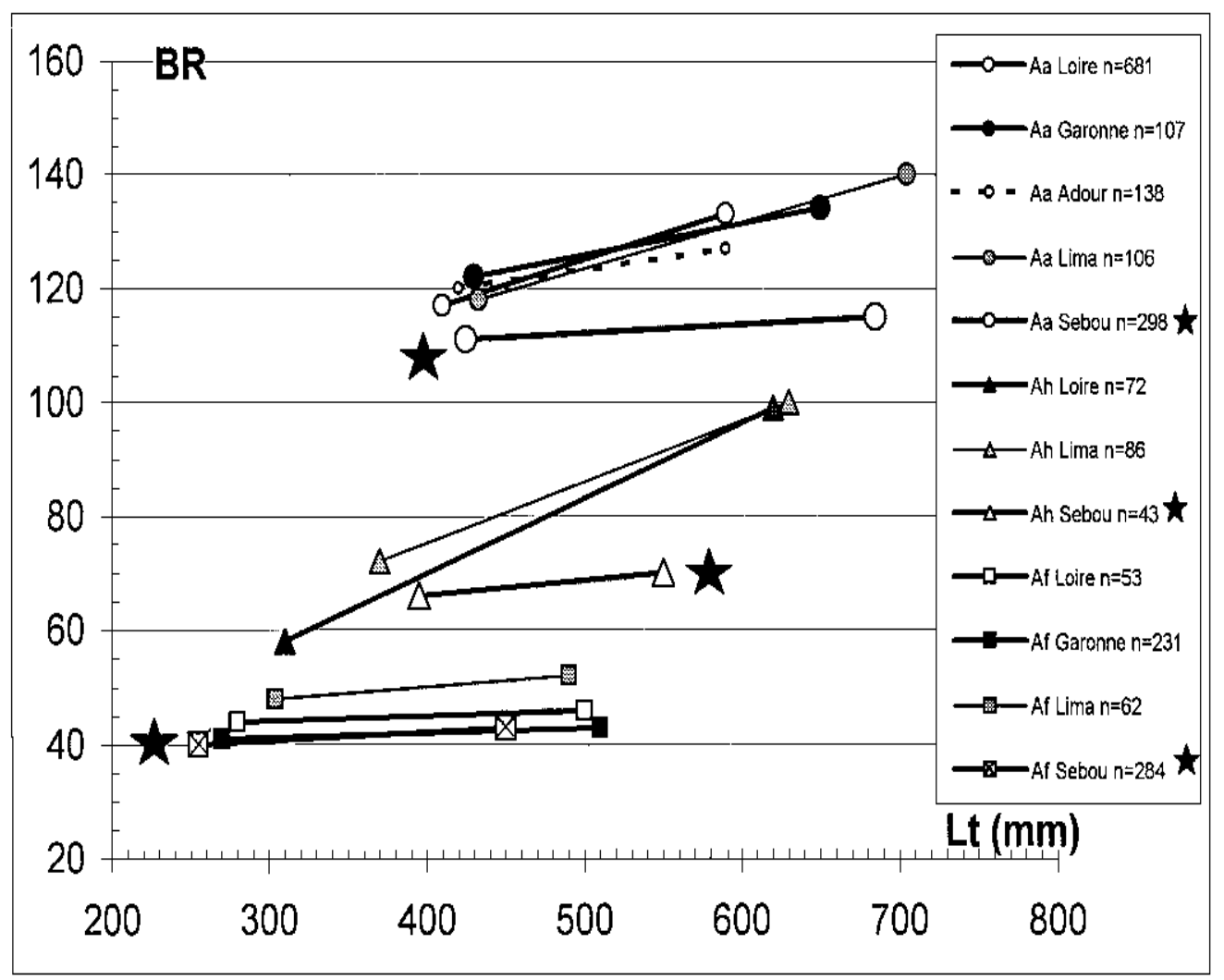

\section{Figure 4}

Aloses vraies, hybrides et feintes, sexes confondus : variations géographiques des régressions du nombre branchiospines $(\mathrm{Br})$ en fonction de la longueur totale (Lt).

\section{Figure 4}

Regression of the geographic variation in total individual gillraker number $(\mathrm{Br})$ in relation to total length (Lt) in both sex of Allis Shad, hybrids and Twaite Shad.

Plusieurs hypothèses peuvent expliquer cette différence touchant un caractère pourtant héréditaire moins sensible que d'autres aux facteurs du milieu (DAGET et BAUCHOT, 1976) :

- une température plus élevée durant l'incubation et favorisant la réduction du nombre d'éléments (HIMBERG, 1976).

- une adaptation liée à la qualité de la nourriture et permettant dans ce cas, par augmentation de la capacité de filtration, de mieux exploiter les proies épaisses (O'MAOILEIDIGH et al., 1988 ; LINDSEY, 1981) présentes probablement dans la zone d'upwelling. Des spécificités biologiques remarquables ont été soulignées par rapport aux autres populations atlantiques par divers caractères concernant la méristique, l'âge, la croissance et la migration (SABATIÉ, 1990,1993 ; SABATIÉ et al., 1996) (Figure 1).

- une compétition alimentaire forte entre les espèces planctonophages plus abondantes sur ces zones (CURY et FONTANA, 1988 ; LINDSEY, 1981) provoquant le décalage des valeurs moyenne du caractère entre taxons. 


\section{Tableau I}

Comparaison du caractère méristique « nombre moyen de branchiospines 》 chez les grandes aloses et hybrides (fond grisé) des fleuves Loire (BOISNEAU, 1990), Garonne (TAVERNY, 1991), Adour (PROUZET et al., 1994), Lima (ALEXANDRINO, 1996) et Sebou (SABATIÉ, 1993). (F) : test d'égalité des variances ; $(t)$ : test de comparaison des moyennes ; (n.s.) différence non significative ; $\left({ }^{* *}\right)$ significative pour $P<0,01 ; n=$ effectif.

\section{Table I}

Meristic comparison of total gillraker number in Allis Shad and hybrids (foreground grey) from Loire River (BOISNEAU, 1990), Garonne River (TAVERNY, 1991), Adour River (PROUZET et al., 1994), Lima River (ALEXANDRINO, 1996) et Sebou River (SABATIÉ, 1993). (F) : one way analysis of variance ; $(t)$ : t-test ; (n.s.) difference non significant ; $\left(^{* *}\right)$ significant for $\mathrm{P}<0,01 ; \mathrm{n}=$ effectif number.

\begin{tabular}{|c|c|c|c|c|}
\hline & Loire & Garonne & Adour & Lima \\
\hline \multicolumn{5}{|l|}{ Loire $n=681$} \\
\hline \multicolumn{5}{|l|}{ Loire $n=72$} \\
\hline Garonne $n=331$ & $F=1,83^{* *}: \quad t=* *$ & & & \\
\hline Adour $n=138$ & $F=4,65 * \star \quad t=* *$ & $F=2,53 * *: t=n . s$. & & \\
\hline Lima $n=106$ & $F=1,10$ n.s : $t=* \star$ & $F=1,66 \quad * *: \quad t=* *$ & 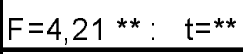 & \\
\hline Lima $n=86$ & $F=2,15^{* *}: t=* *$ & & & \\
\hline Sebou $n=303$ & $F=2,25^{* *}: t=* *$ & $F=1,22$ n.s. : $t=\star \star *$ & $F=2,06^{\star \star}: \quad t=\star \star$ & $F=2,04$ ** : $t=* *$ \\
\hline Sebou $n=47$ & $F=1,97^{* *}: t=* *$ & & & $F=4,24^{* *}:$ \\
\hline
\end{tabular}

Par ailleurs, si l'attention se porte sur l'ensemble des éléments méristiques, non présenté ici, on peut observer l'existence d'un gradient rudimentaire décroissant des valeurs moyennes de plusieurs caractères (rayons de nageoires, scutelles, vertèbres) sans qu'il y ait toutefois un cline latitudinal bien prononcé (SABATIÉ, 1993).

Cette observation peut être interprétée comme l'illustration éventuelle chez l'alose marocaine de la conservation de caractères morphologiques primitifs (BEN SALEM, 1988) que l'on peut relier d'ailleurs à une autre hypothèse : les eaux marocaines auraient été une zone refuge au Pleistocène pour ces populations qui seraient la souche phylogénétique dont dérivent les populations plus septentrionales. Cette hypothèse (SABATIÉ, 1993), semble être confirmée par l'étude génétique électrophorétique récente des aloses feintes marocaines (VÉRON, 1999) qui présentent une forte similarité en terme de distance génétique avec les aloses feintes de la méditerranée dont elles seraient issues (Figure 5). 


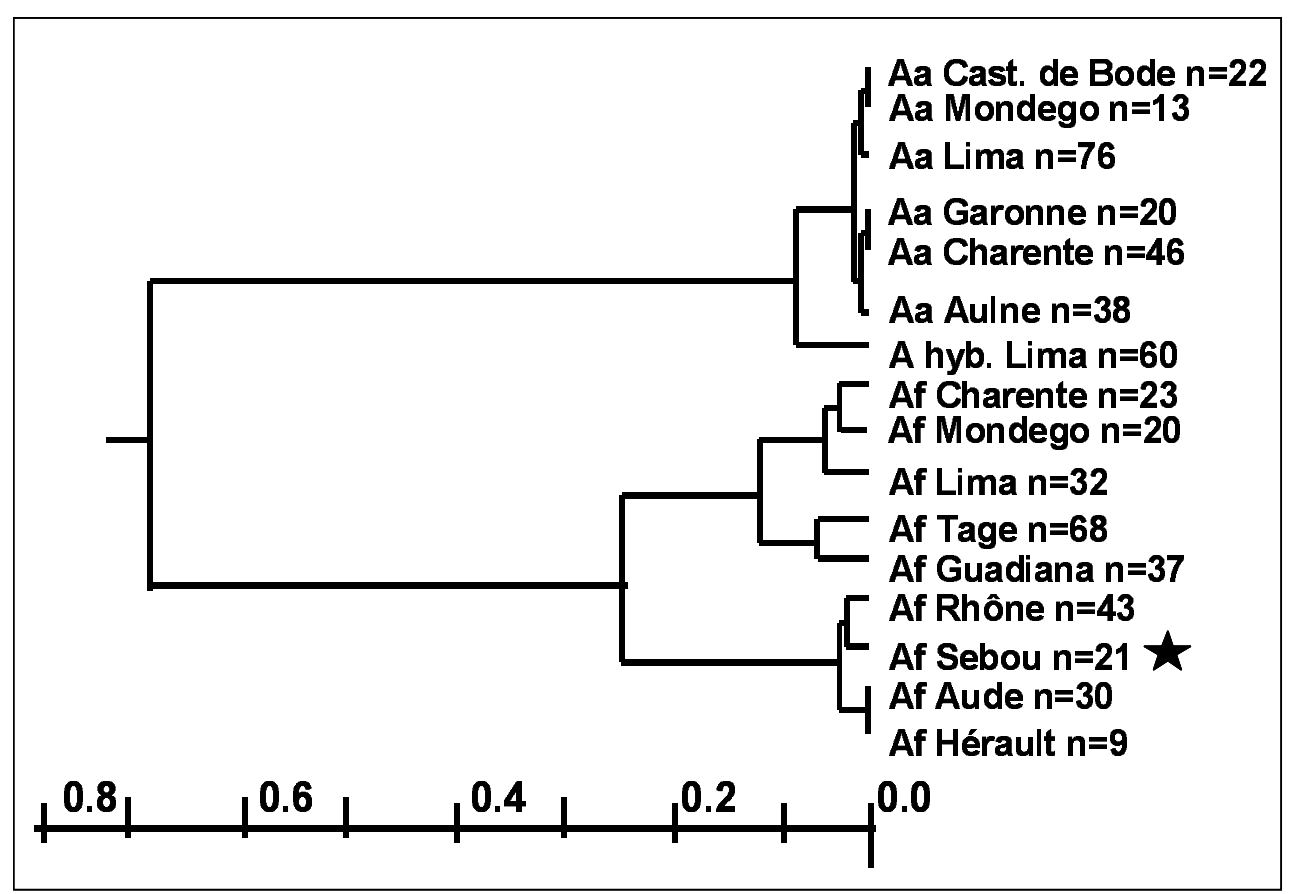

Figure 5

Dendrogramme des distances génétiques entre les populations d'aloses vraies, hybrides et feintes des côtes de l'Atlantique-Est et de la Méditerranée occidentale (matrice des distances génétiques de Nei - 5 loci : ADA, ADH, HBA, MPI, PX méthode UPGMA (unweighted pair-group method with arithmetic averaging ; $\mathbf{n}=$ effectif)).

Figure 5

Dendrogram of genetic distances between Allis Shad, hybrids and Twaite Shad populations from eastern atlantic coasts and western Mediterranean Sea (matrix of Nei's genetic distances ; 5 loci : ADA, ADH, HBA, MPI, PX -UPGMA method (unweighted pair-group method with arithmetic averaging; $n=$ sample size)).

\section{Age}

Les grandes aloses marocaines offrent une large gamme d'âge ( 3 à 8 ans) où prédominent les âges 5 et 6 ans (Figure 6), ce qui est un caractère général observé sur l'ensemble de la façade atlantique (MENESSON-BOISNEAU et al., 2000). Toutefois, la particularité réside dans la difficulté à interpréter les écailles (comme les otolithes) qui portent des marques marines hivernales cicatricielles (Figure 7) et la présence chez $21 \%$ des alosons d'une marque de crue automnale liée au stress supporté durant les violentes crues qui les expulsent vers l'aval (Figure 8). De plus, aucune marque de ponte n'a été constatée chez la grande alose comme étonnamment chez l'alose feinte. 


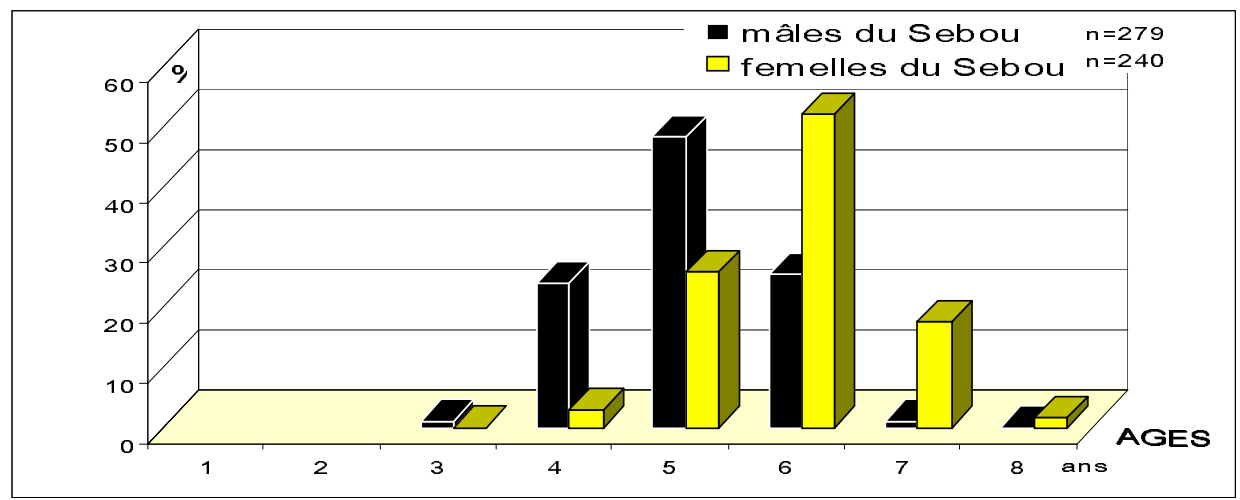

Figure 6

Structure en âge des aloses vraies du Sebou.

\section{Figure 6}

Age distribution in Allis Shad from Sebou River.

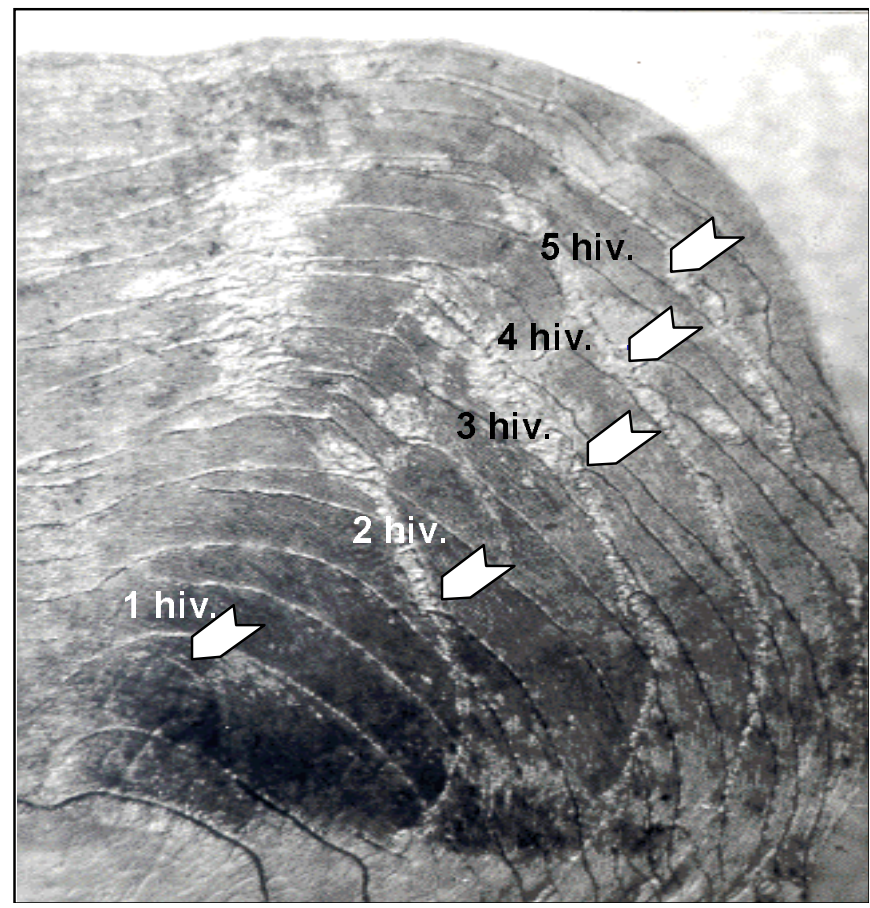

\section{Figure 7}

Ecaille d'une alose vraie femelle $(\mathrm{Lt}=66 \mathrm{~cm})$ capturée un premier mars dans le Sebou ; la cicatrisation des marques hivernales est caractéristique.

\section{Figure 7}

Scale from female Allis Shad caught on March 1 in Sebou River $(L t=66 \mathrm{~cm})$; cicatricial winter rings are characteristic. 


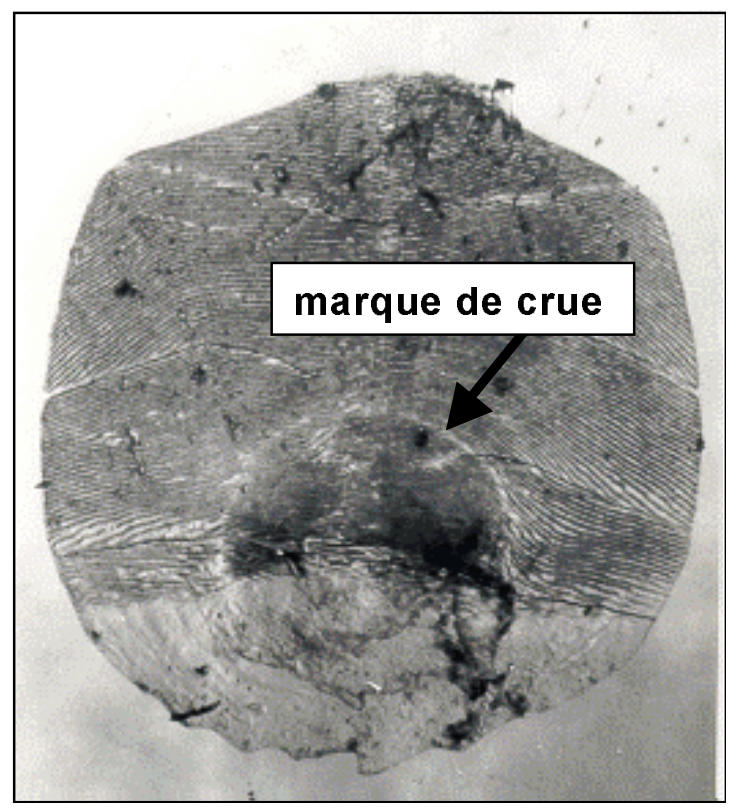

\section{Figure 8}

Ecaille d'une alose vraie juvénile capturée un 10 mai dans le Sebou $(L t=14 \mathrm{~cm})$; présence d'une marque de crue typique.

\section{Figure 8}

Scale from juvenile Allis Shad caught on May $10(L t=14 \mathrm{~cm})$; presence of a flood mark.

Cette illisibilité des structures et leur interprétation délicate résultent probablement de l'instabilité hydroclimatique de l'écosystème marin en zone d'upwelling (...anomalies thermiques contrastées des eaux côtières) et de son influence sur la formation des annuli. Durant la phase continentale l'hydrodynamisme contrasté des oueds au cours des saisons pertube aussi certainement la régularité des formations minérales constatées dans les écailles ou les otolithes (OMBREDANE et BAGLINIĖRE, 1992).

\section{Croissance}

La grande Alose marocaine, comme celle des côtes portugaises (SABATIÉ et al., 1996), présente des performances de croissance en longueur et en poids supérieures à celles des autres populations atlantiques (Figures 9, 10).

Ces croissances résultent de la richesse alimentaire dont bénéficient les juvéniles durant leur dévalaison et les adultes sur les aires productives de l'upwelling localisé entre Essaouira et Agadir (Figure 1). En revanche, chez les aloses feintes qui demeurent à proximité des embouchures, ces performances particulières n'apparaissent pas. 


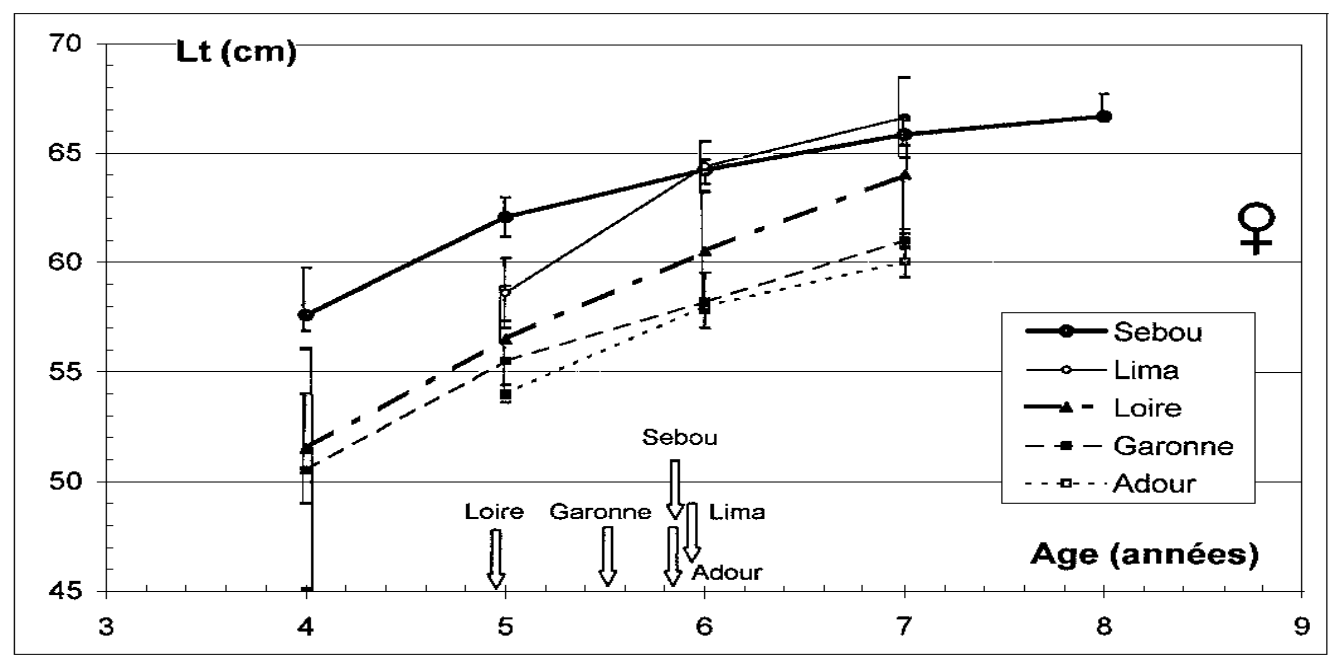

Figure 9

Croissance comparée moyenne en longueur des aloses vraies femelles (fleuves Loire, Garonne, Adour, Lima et Sebou). Les âges moyens sont fléchés.

\section{Figure 9}

Growth comparison in length between female Allis Shad of different populations (Loire, Garonne, Adour, Lima and Sebou rivers) ; average ages are marked with arrow.

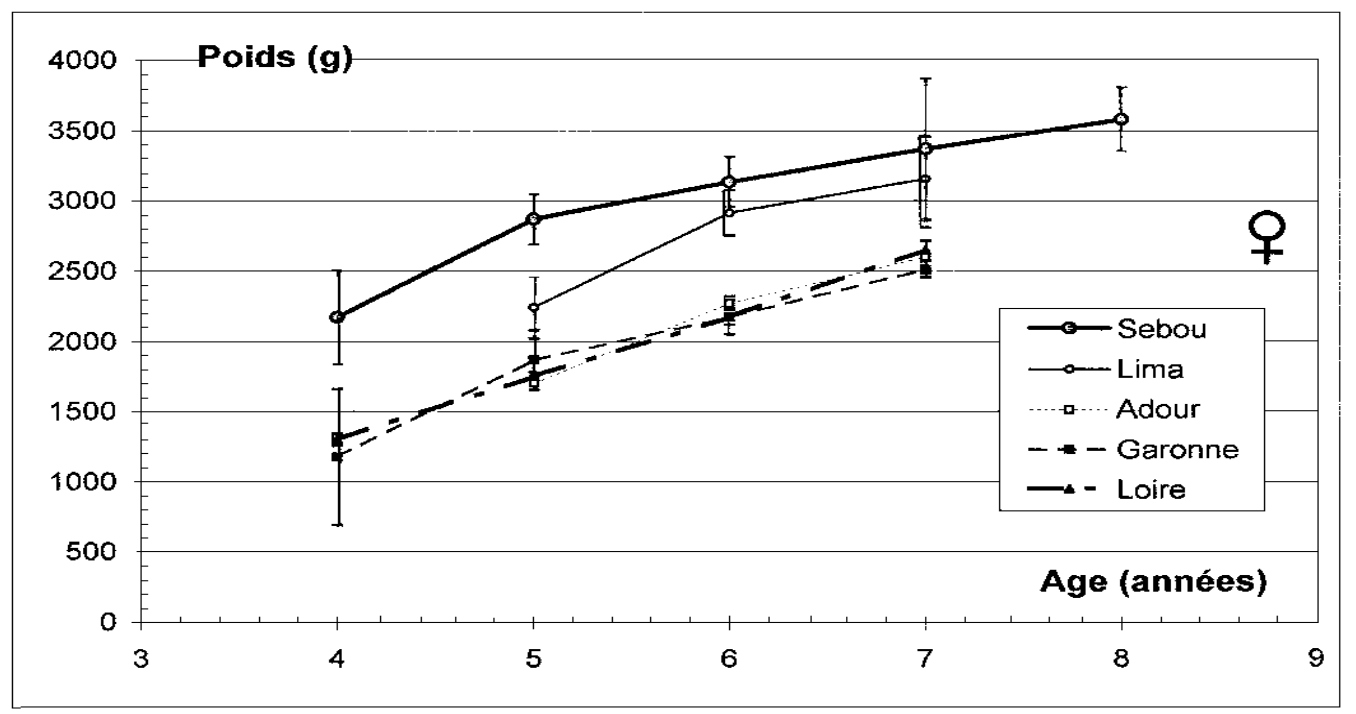

Figure 10

Croissance comparée moyenne en poids des aloses vraies femelles (fleuves Loire, Garonne, Adour, Lima et Sebou).

\section{Figure 10}

Growth comparison in weight between female Allis Shad of different populations (Loire, Garonne, Adour, Lima and Sebou rivers). 


\section{Migration}

La population d'aloses vraies du Sebou effectue un cycle migratoire qui la conduit des zones de frai vers une zone d'engraissement localisée à proximité de l'upwelling localisé entre Essaouira et Agadir (SABATIÉ, 1993) (Figure 1). Par rapport aux fleuves septentrionaux on peut constater que le flux migratoire est beaucoup plus étalé dans le temps (6 mois) et que le début de migration est plus précoce de trois mois (SABATIÉ et al., 1996) (Figure 11).

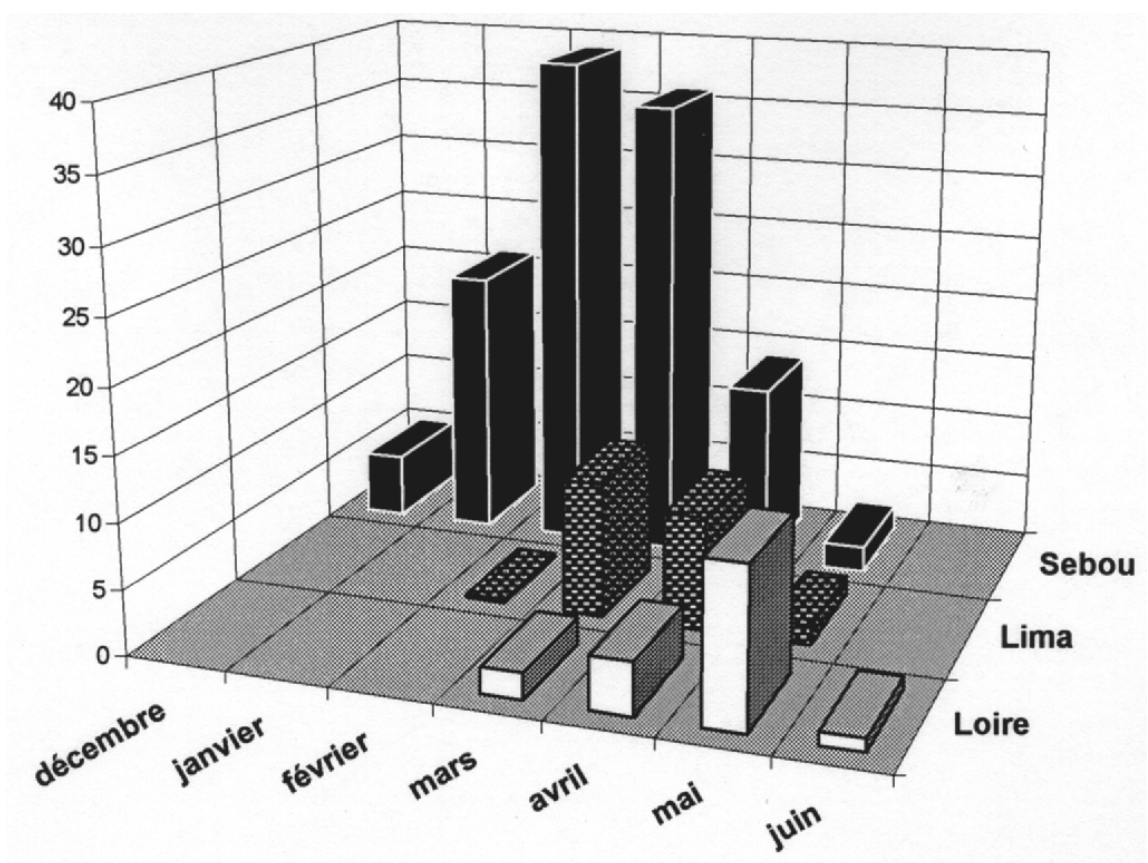

\section{Figure 11}

Captures mensuelles d'aloses vraies dans les fleuves Sebou, Lima et Loire ; évolution latitudinale des périodes de migration.

\section{Figure 11}

\section{Latitudinal development of the period of migration according to monthly catches of Allis Shad from Sebou, Lima and Loire Rivers.}

Ces particularités peuvent être consécutives au décalage clinal des températures moyennes des eaux sur la façade atlantique et, dans le Sebou, aux débits élevés qui apparaissent en automne après 7 à 9 mois de sécheresse. Ceux ci stimulent alors la migration génésique comme cela peut être observé au printemps à proximité des fleuves méditerranéens qui subissent aussi de fortes fluctuations des débits (RAMEYE et al., 1976 ; DOUCHEMENT, 1981 ; LE CORRE, 1999). La durée relativement plus allongée du cycle pourrait être reliée aux divers comportements de diffusion qui conduisent les aloses à parcourir une distance importante entre la zone d'upwelling et l'embouchure du Sebou.

\section{CONCLUSION}

L'étude de divers caractères concernant la méristique, l'âge, la croissance et la migration (SABATIÉ, 1993 ; SABATIÉ et al., 1996, 2000 ; MENESSON-BOISNEAU et al., 2000) a souligné des spécificités biologiques remarquables par rapport à celles des autres populations atlantiques. 
Les populations d'aloses du Maroc et l'alose vraie en particulier, représentaient une haute valeur socioculturelle et une activité de pêche saisonnière lucrative qui a perduré jusqu'à l'année 1929. Cette date marque le début des constructions de barrages non équipés de passes à poissons fonctionnelles et la progression de diverses pollutions. Par la suite, la pêche mal gérée (voir l'exemple du Sebou, Figure 2), a rapidement entraîné le déclin de l'espèce $A$. alosa qui a disparu des eaux marocaines depuis 1992.

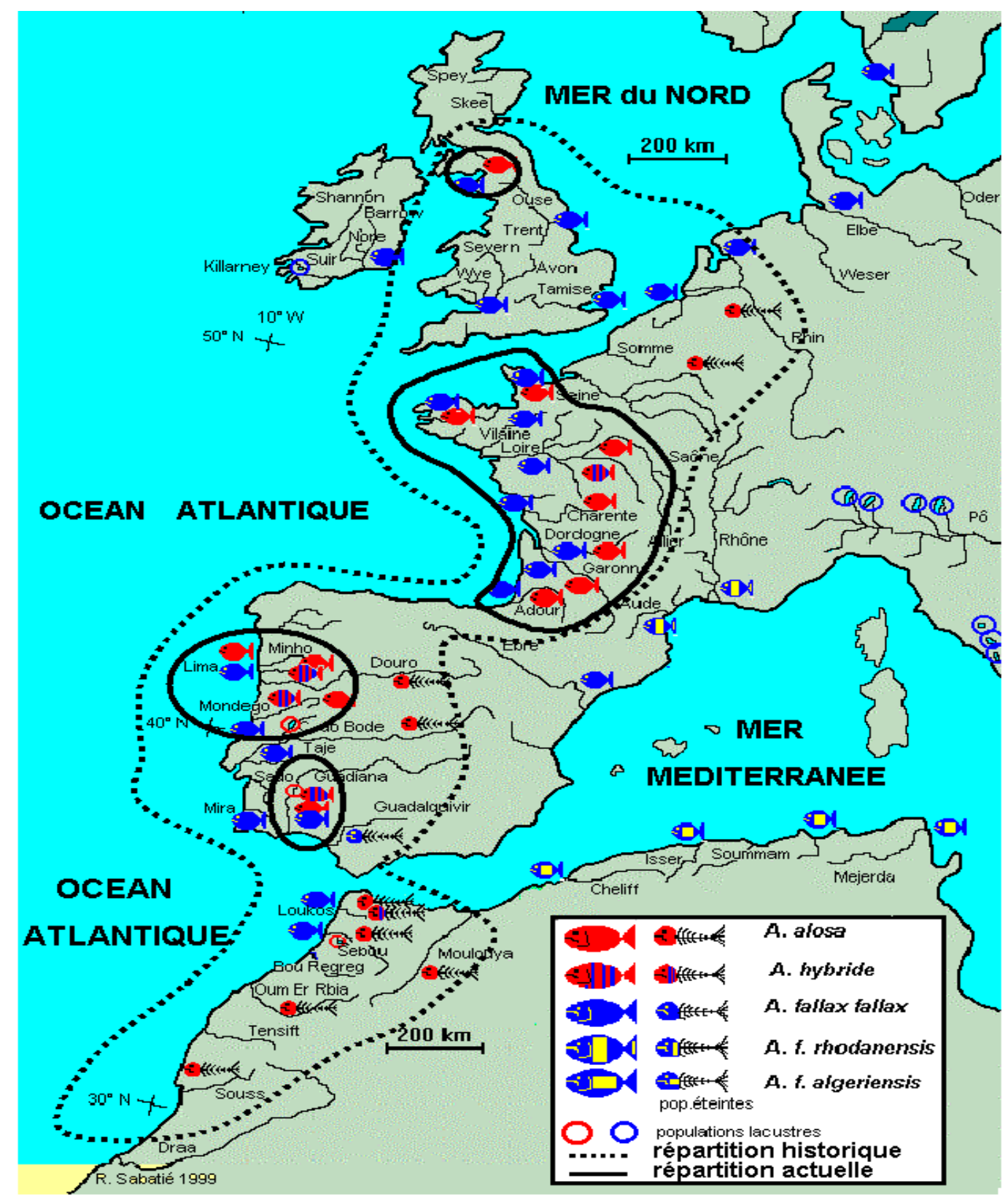

Figure 12

Aire de distribution ancienne et actuelle des aloses vraies le long des côtes atlantique-est.

\section{Figure 12}

Historic and current distribution area of Allis Shad populations along the Eastern Atlantic coasts ; the symbols indicate the current status of the sub-populations. 
Cette disparition fragmente davantage l'aire de distribution dans sa partie sud (Figure 12) et la réduit de presque $1200 \mathrm{~km}$ vers le nord! Cette réduction pourrait à l'avenir être encore plus forte lorsque l'on considère l'état de certaines populations portugaises. L'alose feinte est encore présente sur le long des côtes marocaines, même si son avenir semble également bien menacé façe aux abus des usages et de la gestion de l'eau.

Bien qu'elle offrait d'intéressantes perspectives de recherches, l'alose vraie a malheureusement disparue avant qu'il fût possible d'analyser son patrimoine génétique dont la connaissance était fondamentale pour reconstruire l'histoire phylogénétique de ce taxon. II est désormais trop tard pour tenter d'entreprendre des actions de restauration sur cette espèce même si une volonté se dessine pour améliorer la libre circulation des migrateurs.

La disparition de cette espèce qui pouvait être un symbole pour le Maroc dans la maîtrise de la conservation de la biodiversité ichtyologique, montre l'incohérence de la gestion d'une ressource exploitable et renouvelable recouvrant un espace côtier et continental.

\section{BIBLIOGRAPHIE}

ALEXANDRINO P., 1996. Estudo de populaçoes de savel (Alosa alosa L.) e savelha (Alosa fallax Lacépède). Analise da diferenciaçao interspecifica, subestruturaçao e hibridaçao. Thèse de Doctorat, Université de Porto. $185 \mathrm{p}$.

AZEROUAL A., CRIVELLI A., DAKKI M., YAHYAOUI A., 2000. L'ichtyofaune des eaux continentales du Maroc. Com. affichée. Rencontres Ichtyologiques Françaises (RIF) 2000, Cybium, (à paraître).

BEN SALEM M., 1988. Taxinomie numérique des espèces du genre Trachurus Rafinesque 1810 (Poissons Téléostéens Carangidae). Cybium, 12 (1), 45-59.

CURY P., FONTANA A., 1988. Compétition et stratégies démographiques comparées de deux espèces de sardinelles (Sardinella aurita et Sardinella maderensis) des côtes ouest-africaines. Aquat. Living Resour., 1, 165-180.

DAGET J., BAUCHOT M.L., 1976. Les problèmes de l'espèce chez les téléostéens. Mém. Soc. Zool. de France, 38, 67-117.

DOUCHEMENT C., 1981. Les aloses des fleuves français Alosa fallax Lacépède 1803 et Alosa alosa Linné 1758. Biométrie écobiologie autonomie des populations. Thèse de Doctorat $3^{\text {ème }}$ cycle, Ecol. gén. app., U.S.T.L. Montpellier I, 377 p.

HIMBERG M., 1976. The geographical variation in the number of gillrakers of coregonid fishes in relation to environnemental factors. Rev. Trav. Inst. Pêches marit., 40 (3-4), 602-603.

LE CORRE M., 1999 : Eco-biologie et génétique des populations d'aloses (Alosa fallax spp.) de la Méditerranée française. Thèse de doctorat, option Halieutique, ENSAR Rennes, 189 p. + annexes.

LINDSEY C.C., 1981. Stocks are cameleons : plasticity in gill rakers of coregonid fishes. Can. J. Fish. Aquat. Sci., 38 (12), 1497-1506.

MENESSON-BOISNEAU C., APRAHAMIAM M.W., SABATIÉ M.R., CASSOU-LEINS J.J., 2000. Biologie des aloses : Remontée migratoire des adultes. In: «Les aloses (Alosa alosa et Alosa fallax spp.) : Biologie, écologie, taxinomie et influence des activités humaines ». BAGLINIĖRE J.L. et ELIE P. Eds., INRA-CEMAGREF ed., Paris, 55-73.

O'MAOILEIDIGH N., CAWDERY S., BRACKEN J.J., FERGUSON A., 1988. Morphometric, meristic and electrophoretic analyses of two irish populations of twaite shad, Alosa fallax (Lacépède). J. Fish. Biol., 32, 355-366.

OMBREDANE D., BAGLINIĖRE J.L., 1992. Les écailles et leurs utilisations en écologie aquatique. In : «Tissus durs et âge individuel des vertébrés », Colloque national, 
Bondy, France, 4-6 mars 1991. BAGLINIÈRE J.L., CASTANET J., CONAND F., MEUNIER F.J. (Eds.). Colloques et Séminaires ORSTOM-INRA : 151-193.

PROUZET P., MARTINET J.P., CUENDEC F.X., 1994. Les pêches professionnelles dans les estuaires de la Loire et de l'Adour. Rep. Océans, 6, 31-78.

RAMEYE L., KIENER A., SPILLMANN C.P., BIOUSSE J., 1976. Aspects de la biologie de l'alose du Rhône. Pêche et difficultés croissantes de ses migrations. Bull. Fr. Pêche Piscic., 263, 50-76.

SABATIÉ M.R., 1990. Croissance de l'alose vraie (Alosa alosa L.) dans l'oued Sebou (façade atlantique du Maroc). Cybium, 14 (2), 131-142.

SABATIÉ M.R., 1993. Recherches sur l'écologie et la biologie des aloses au Maroc (Alosa alosa L. 1758 et Alosa fallax Lac. 1803). Exploitation et taxinomie des populations atlantiques. Bioécologie des aloses de l'oued Sebou. Thèse de Doctorat de l'Université de Bretagne Occidentale en Océanologie Biologique. 326 p. + annexes.

SABATIÉ M.R., ALEXANDRINO P., BAGLINIĖRE J.L., 1996. Comparaison des caractéristiques biologiques des aloses vraies (Alosa alosa) dans l'oued Sebou (façade nord-atlantique du Maroc) et dans le feuve Lima (Portugal). Cybium, 20 (3), 59-75.

SABATIÉ M.R., BOISNEAU Ph., ALEXANDRINO P., 2000. Variabilité morphologique et diversité génétique chez les aloses. In: "Les aloses (Alosa alosa et Alosa fallax sp.): Biologie, écologie, taxinomie et influence des activités humaines ». BAGLINIĖRE J.L. et ELIE P. Eds., INRA-CEMAGREF ed., Paris, 137179.

TAVERNY C., 1991. Contribution à la connaissance de la dynamique des populations d'aloses (Alosa alosa et Alosa fallax) dans le système fluvio-estuarien de la Gironde; Pêche, biologie, écologie ; Etude particulière de la dévalaison et de l'impact des activités humaines. Thèse de doctorat de l'Université de Bordeaux 1, 4, 568 p. + annexes.

VÉRON V., 1999. Les populations de grande alose (Alosa alosa L.) et d'alose feinte (Alosa fallax, Lacépède) des petits fleuves français du littoral Manche Atlantique. Mémoire DAA Option Halieutique : ENSA-INRA, Rennes, 79 p. + annexes.

WATIER C., 1918. La pêche de l'alose au Maroc. Ann. Univ. de Grenoble, 29 (3), 5-27. 
